\title{
MS21-05 | Spin-Resolved Atomic Orbital Model Refinement for Combined Charge and Spin Density Analysis: Application to the Perovskite
}

Lecomte, Claude (Universite de Lorraine, Vandoeuvre-les-Nancy, FRA)

A new model was developed in order to refine spin-resolved atomic orbitals from combined charge and spin density studies. This new algorithm allows the simultaneous refinement against $x$-ray diffraction and polarized Neutron diffraction data, giving access to spin-resolved electron densities. This atomic orbital model is applied to the perovskite. The radial Extension, orientation and population of outer atomic orbitals for each atom are calculated. The interaction term between $\mathrm{Ti}^{3+}, \mathrm{Y}^{3+}$ cations and $\mathrm{O}_{2^{-}}$ligands is estimated. A comparison of the refinement qualities obtained by means of the orbital and multipole models, respectively, is presented. 